\title{
Comparison of Different Control Schemes for Strategic Departure Metering
}

\author{
Husni Idris and Ni Shen \\ TASC an Engility Company \\ Billerica, MA, USA \\ husni.idris@engilitycorp.com
}

\author{
Aditya Saraf and Jason Bertino \\ ATAC Corporation \\ Santa Clara, CA, USA \\ aps@atac.com
}

\author{
Shannon Zelinski \\ NASA Ames Research Center \\ Moffett Field, CA, USA \\ shannon.j.zelinski@nasa.gov
}

\begin{abstract}
Airports and their terminal airspaces are key choke points in the air transportation system causing major delays and adding to pollution. A solution aimed at mitigating these chokepoints integrates the scheduling of runway operations, flight release from the gates and ramp into the airport movement area, and merging with other traffic competing for downstream airspace points. Within this integrated concept, we present a simulation-based analysis of the departure metering process, which delays the release of flights into the airport movement area while balancing two competing objectives: (1) maintaining large enough queues at the airport resources to maximize throughput and (2) absorbing excess delays at the gates or in ramp areas to save on fuel consumption, emissions, noise, and passenger discomfort. Three metering strategies are compared which respectively attempt to control the number of flights that (1) left the gate but did not take off, (2) left the ramp but did not take off, and (3) spent their unimpeded transit time to the runway but did not take off. It was observed that under deterministic and demand uncertainty conditions, the first strategy performed better than the other two strategies in terms of maintaining the runway throughput while transferring a significant average delay of two minutes to the gate. On the other hand, under uncertainties of flight transit time and runway service rate, all the strategies struggled to delay flights at the gate without a significant impact on the runway throughput.
\end{abstract}

Keywords-Air Traffic Management; Airport Operations; Integrated Arrival Departure Scheduling; Departure Metering;

\section{INTRODUCTION}

Delays originate mostly at major airports, and particularly at ones that constitute complex metroplexes of multiple interacting airports [1]. Major causes of delay at these metroplex systems are constrained resources on the airport surface, particularly the runways and in the surrounding terminal airspace. These complex systems are characterized by high levels of interaction between flows of multiple adjacent airports sharing arrival and departure fixes and competing for gaps in the overhead traffic streams. Solutions to mitigate these choke points have consisted mostly of isolated concepts and capabilities that are applied to components of the system. For example, concepts have focused on arrival flow management for arrival metering, sequencing and spacing [2, 3], on departure flow management such as departure metering at gates [4-6] and precision departure release control [7, 8], or on runway scheduling $[9,10]$. Integrated solutions are needed in order to reap the benefits of the isolated capabilities [11]. Research has been conducted to demonstrate integrating arrival operations from the en route phase of flight to the landing [12]. Initial research into integrating runway arrival and departure operations has also been conducted, generating preliminary concepts and algorithms [13-15]. In this paper, we describe a research activity that supports a NASA Advanced Technology Demonstration (ATD-2) project aimed at integrating departure and arrival operations in a metroplex, with an emphasis on integrating departure operations within arrival constraints [1617].

The integration of airspace and surface operations in a metroplex system aims to maintain high throughput of the system, efficient operations through expedited, uninterrupted movement, and minimum fuel burn and emission. This integration attempts to achieve these objectives through in part the generation of a coordinated schedule of departures at key resources or control points. These control points include primarily the release from the gates, the exit from the ramp area into the airport movement area, the takeoff from the runways, and the crossing of departure fixes, as depicted in Fig. 1. It may be possible to simultaneously achieve these objectives under deterministic conditions. However, uncertainty in the operations and the environment brings about tradeoffs among them. For example:

1. In order to maintain high throughput under uncertainty, delay or queue buffers are needed to keep pressure on the choked resources of the system and take advantage of any service opportunities that may arise. Parallel queues provide controllers the ability to sequence departures optimally and the controllability needed for conformance to prescribed constraints such as flow management restrictions. If the

\footnotetext{
This research was funded by NASA under contract NNA14AB46C.
} 
schedule assumed the fastest transit between resources with zero delay or queue buffers it would be violated due to any disturbance that results in longer transit time. This requires absorbing some delay near downstream resources, such as in the airspace near departure fixes and on the airport surface near the runways, as shown in Fig. 1.

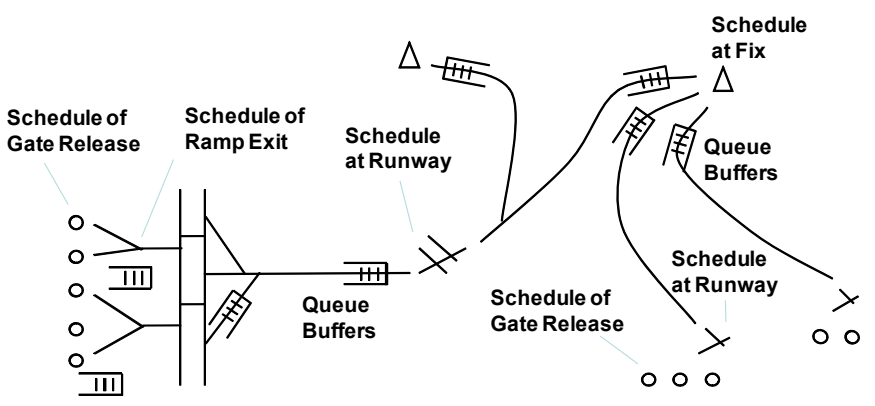

Fig. 1. Integrated scheduling with target queue buffers

2. On the other hand, delay is absorbed more efficiently and cleanly on the airport surface (movement area) rather than in the airspace, and in turn, at the gate while the engines are off rather than on the airport surface. Absorbing delay at the gates or in the ramp area saves on fuel consumption, emissions and noise in addition to allowing passengers to absorb some of the delay more comfortably while off the plane.

Therefore, the integrated scheduler needs to decide on the distribution and allocation of delay between the interconnected resources. NASA's ATD-2 concept includes a strategic surface scheduler which schedules the release of flights from the gates and/or the ramp when the demand for the runways is predicted to exceed their capacity in order to balance the tradeoff between throughput and efficiency. Key parameters used in this decision are the desired queue or delay buffer sizes that the scheduler should target to achieve an appropriate balance between these objectives.

We present a simulation-based analysis of the departure metering process. Three metering strategies that are being considered for the ATD-2 strategic scheduling applied at Charlotte International Airport (CLT) are compared. They respectively attempt to control the number of flights that (1) left the gate but did not take off, (2) left the ramp but did not take off, and (3) spent their unimpeded transit time to the runway but did not take off. We draw insights on the performance of these strategies in terms of conforming to the runway schedule while transferring delay to the gates and ramp area. We assess the performance of dynamic metering under different scenarios of uncertainty about the demand, the flight transit time and the runway schedule.

In the following sections we describe the ATD-2 operational concept and assumptions made for this analysis. Then, we describe the fast-time simulation model that was developed and used for this analysis, along with the underlying algorithms and statistical models. The analysis results are then reported with insights on the performance of the different metering strategies under different uncertainty scenarios, ending with concluding remarks and future extensions.

\section{ATD-2 CONCEPT AND ASSUMPTIONS}

The ATD-2 concept includes several attributes that achieve the integration between the scheduling of operations at different resources. Two of these attributes are:

1. Integration between upstream and downstream schedules. Namely, the schedule of operations at the runway considers constraints stemming from scheduling flows at downstream airspace shared points such as TRACON departure gates and gaps in the overhead en route stream. In turn, the schedule of the release from the gates or ramp area into the airport movement area takes into consideration the restrictions stemming from the schedules at the runways and at downstream airspace merge points.

2. The concept integrates a strategic metering scheduler with a tactical control scheduler. The strategic scheduler implements the metering process; it runs at low frequency and attempts to control the congestion at reasonable levels when demand exceeds capacity. The tactical scheduler runs more frequently and controls the conformance of the flights to the integrated schedules at the different resources such as the runway and the release from the ramp and gates into the airport movement area.

The interactions between the upstream and downstream schedulers and between the strategic and tactical schedulers are a topic of continued research and design. For this analysis, we made some assumptions about these interactions, as described in the following paragraphs of this section.

The strategic scheduler implements a metering process by generating a desired schedule of releases from the gates and ramp area such that congestion is reduced and a desired level of queuing buffers is maintained. The queuing may be measured and controlled using different parameters, three of which are compared in this paper: (1) the number of flights that left the gate but did not take off (2) the number of flights that left the ramp area but did not take off and (3) the number of flights that have spent their unimpeded transit time since they left the gate or the ramp to the runway but did not take off. These three queuing parameters correspond to the number of flights in three queuing systems of different sizes. The exit from all three systems is the takeoff event. The entry to the first system is the pushback, to the second system is the exit from the ramp into the movement area, and to the third system is when the flight's unimpeded transit time since its pushback or its ramp exit has elapsed. The first two systems are physical while the third system is virtual because the elapsed unimpeded transit time does not correspond to a physical resource or location in the airport. The first system is the largest containing more flights, followed by the second system. Finally the third system is the smallest and attempts to approximate the number of flights that are closer to the runway end.

The strategic scheduler requires a runway schedule in order to estimate the level of congestion and queuing based on the demand. Rather than generating its own runway schedule, it is assumed in this analysis that the strategic scheduler takes the 
most recent runway schedule generated by the tactical scheduler as an input. Therefore, the tactical scheduler was given the same time horizon as the strategic scheduler in this analysis; otherwise the strategic scheduler would need to extend the tactical schedule to a longer horizon. The tactical schedule ensures the separation requirements between successive runway operations and integrates restrictions stemming from downstream schedules at departure fixes and en route overhead merge points, and possibly at arrival metering points and destination airports. In this paper, the model used for the tactical scheduler (described in the next section) applies the separation requirements; however, it does not explicitly take the other restrictions into consideration. A prior paper described an algorithm for this integration of downstream schedules into the runway schedule [28].

The strategic scheduler generates desired release times from the gates to control the queuing parameter while ensuring that the input runway schedule remains feasible. The flight release time from the gate ensures that the flight can transit unimpeded to the runway in time to make its assigned runway time, even if the queuing parameter exceeded the desired threshold. In this case the queuing parameter may exceed the desired level in favor of enabling the runway schedule to be feasible. Alternative schemes that are not analyzed in this paper include the strategic scheduler giving priority to maintaining the desired queuing buffers over conformance to the runway schedule. In this case the strategic scheduler may recommend gate release times that maintain the queuing parameter at or below the desired level but require changes to the input runway schedule. The strategic scheduler can suggest the changes in the runway schedule that correspond to the recommended gate release times. However, ultimately the tactical scheduler determines the final runway schedule and the corresponding gate release time reconciling the strategic scheduler recommendations with other constraints and objectives.

The tactical scheduler has the task of ensuring the conformance of flights to the runway schedule and to the releases from the gate and ramp into the movement area recommended by the strategic scheduler. It has to make a decision if the two schedules cannot be met simultaneously. In actual operations, this decision should be mostly resolved in favor of meeting the runway schedule to maintain the throughput of the runway bottleneck at the expense of exceeding the desired queuing buffers. However, in this analysis we assume that the tactical scheduler adheres to the desired release times recommended from the strategic scheduler in order to measure the performance of the strategic scheduler in terms of enabling the conformance to the runway schedule. Otherwise the tactical scheduler would override the release times computed by the strategic scheduler.

The strategic scheduler takes into account capacity constraints at the gates by ensuring that the departures are not delayed excessively such that arriving flights requiring the gate incur delay. The strategic scheduler may not have access to full information about the airline gate capacities and procedures hence this constraint needs to be approximated by the strategic scheduler. In this analysis we assumed that the available gates are pooled into one set of gates that are used by all users. The model is capable of limiting the gate capacity by users and aircraft types, but this capability was not exercised in this analysis.

The strategic scheduler takes as input the demand in terms of expected pushback times over the scheduling horizon. We assume that the strategic scheduler knows the actual push back times and the actual ramp exit times for the flights that have already pushed back or exited the ramp, respectively. For flights that have not pushed back yet, it assumes the published pushback time in the flight plan. For flights that have not exited the ramp yet, it assumes a scheduled ramp exit time estimated by adding an unimpeded transit time from the gate to the ramp to the flight plan pushback time. As a baseline for comparison, we assume that the pushback and exit times are known by the scheduler by using the actual pushback and ramp exit times instead of the pushback time published in the flight plan and its corresponding estimated ramp exit time.

\section{MODELS AND AlgORITHMS}

A fast time simulation was used for the analysis of the ATD-2 concept elements. The fast time simulation implements models for the strategic and tactical schedulers and the interactions between them according to the assumptions described in the previous section. The algorithms and underlying statistical models used for the three main components of this simulation are described in the following subsections.

\section{A. Strategic Scheduler}

The strategic scheduler takes a runway schedule as an input and computes gate and ramp release times that maintain a queuing parameter at or below a desired value. Three queuing parameters are compared in this paper: (1) the number of flights that left the gate but did not take off (2) the number of flights that left the ramp area but did not takeoff, and (3) the number of flights that have spent their unimpeded transit time since they left the gate or the ramp to the runway but did not takeoff. As described above, the three parameters correspond to three queuing systems, with different entry events and the same exit event (the takeoff). The entry to the first system is the pushback, to the second system is the ramp exit, and to the third system when the flight's unimpeded transit time to the runway elapses. Henceforth we call the time associated with the entry to any of the systems the entry time. We describe the flights that entered the third system (have spent their unimpeded transit time to the runway since they left the gate or the ramp) as ready for takeoff. This is an approximation as these flights may not be physically ready for takeoff if some of their unimpeded transit time was spent absorbing delay rather than transiting towards the runway. We also call the corresponding entry time to the third system (equal to the unimpeded transit time after leaving the gate or the ramp) the takeoff ready time. First the metering algorithm is described followed by the statistical model used to determine the desired queuing parameter threshold. The input runway schedule generated by the tactical scheduler is described in the next section. 


\section{1) Metering algorithm}

Based on the input runway schedule, the metering algorithm computes three numbers for each flight within its scheduling horizon: the gate release time, the ramp exit time (also called taxi spot release time) and the takeoff ready time. In the first case, controlling the number of flights that pushed back but did not take off, the algorithm first computes the desired push back time and then derives the spot release and takeoff ready times by adding the unimpeded transit times of the flight. In the second case, controlling the number of flights that left the spot but did not takeoff, the algorithm first computes the desired spot release time and then derives the gate pushback time and the takeoff ready time by subtracting and adding (respectively) the unimpeded transit times of the flight. Finally, in the third case, controlling the number of flights that are ready for takeoff but did not takeoff, the algorithm first computes the takeoff ready time and then derives the other two times by subtracting the unimpeded transit times of the flight. The algorithm is the same for the three cases except for considering the entry time to the queuing system as the estimated pushback time, the estimated ramp exit time, or the estimated takeoff ready time, respectively. This time is referred to in the following description as the entry time. The metering algorithm first ranks the flights by the estimated pushback time as a representation of the first come first serve (FCFS) order and then performs the following steps for each flight in this FCFS order:

1. Estimate the queuing parameter value at the estimated entry time of the flight.

2. Determine the release time that satisfies the desired queuing parameter threshold: If the value estimated in step 1 is larger than the target parameter threshold (described in the following subsection), perform the following two steps:

a. Identify the flights that have already entered the system (their entry time is earlier than or equal to the entry time of the flight being scheduled) but have not exited the system (their takeoff time is later than the entry time of the flight being scheduled).

b. Set the desired entry time at the takeoff time of the flight with the earliest takeoff time that reduces the parameter to the target value.

3. Apply the runway schedule conformance constraint if turned on: If the algorithm is set to conform to the runway schedule, set the desired entry time to the minimum of two numbers: (1) the computed value in step 2 or, (2) the flight's schedule takeoff time minus its unimpeded transit time from its entry to the queuing system to the runway. This ensures that the flight is able to transit unimpeded to the runway in time to meet its takeoff schedule. If not set to conform to the runway schedule, the desired entry time from step 2 is unaltered. Note that for the third control strategy that uses the takeoff ready time as the entry time, the runway schedule conformance constraint is always on because the value (1) is never larger than the schedule takeoff time and the value (2) is exactly the schedule takeoff time. In this paper, the runway schedule conformance constraint was always turned on to enable fair comparison between the strategies.

4. Apply the gate constraint if it is enforced: Find the arrivals that arrived at their gate before the flight's candidate gate release time computed in step 3. The gate arrival times used in the model are the actual parking times recorded in the ASPM database (IN times). Estimate the gate demand at each of these times as the sum of arrivals and departures that are, or would still be, at their gate. Set the desired entry time to the earliest value at which the gate demand drops below the threshold gate capacity value. The gate capacity was estimated using historical data analysis as the maximum number of gates that were occupied at the same time, which for CLT was determined to be 97 gates.

5. Apply a maximum position shift if enforced: a constraint is applied on the change between the sequence of the flight at the entry and its sequence in the runway schedule in terms of number of flights that the flight needs to pass between its entry and its takeoff. This constraint reflects the limited ability to re-sequence flights between the entry point (gate, ramp, or runway end) and the takeoff. If the constraint is enforced, first the scheduled takeoff times of the flights that are in the system ahead of the flight are identified (as defined in step 2.a). Then the flight's entry time is set at the earliest of these takeoff times that keeps the required position shift below a threshold. In this analysis this constraint was relaxed by setting the maximum position shift threshold to infinity.

6. Once the desired entry time that satisfies all the active constraints is computed, the other two output times are computed by adding or subtracting the unimpeded transit times of the flight. The statistical models of the unimpeded transit times are described later in this section.

\section{2) Saturation model}

The strategic scheduler requires as a key input the target value of the queuing parameter for each of the three control strategies analyzed. This value was estimated using a throughput saturation analysis of historical data and varied in the analysis to determine the sensitivity of the scheduler performance to it. The throughput saturation models were described in [27]. The historically reported throughput is plotted against the historically reported number of flights in the queuing system as shown in Fig. 2 for one CLT runway (18L) using one year of data (10-1-2011 to 9-30-2012). In this plot, 
the number of flights plotted on the horizontal axis is the first queuing parameter (the number of flights that left the gate but did not take off), which is used as an example to demonstrate the model. The model is applied to all the other queuing parameters in an identical manner but the corresponding plots are not shown. The runway throughput is plotted on the vertical axis and the number of flights in the queuing system, $\mathrm{N}(\mathrm{t})$, is plotted on the horizontal axis. The number of flights $\mathrm{N}$ plotted on the horizontal axis was measured at every minute $t$ as the number of flights that were in the queuing system at that minute - this is the number of departures that have entered the system (their pushback time is less than or equal to $t$ in this case) but have not exited from the system (their takeoff time is larger than $t$ ) by that minute. The entry time was set differently for each of the definitions of the queuing systems.

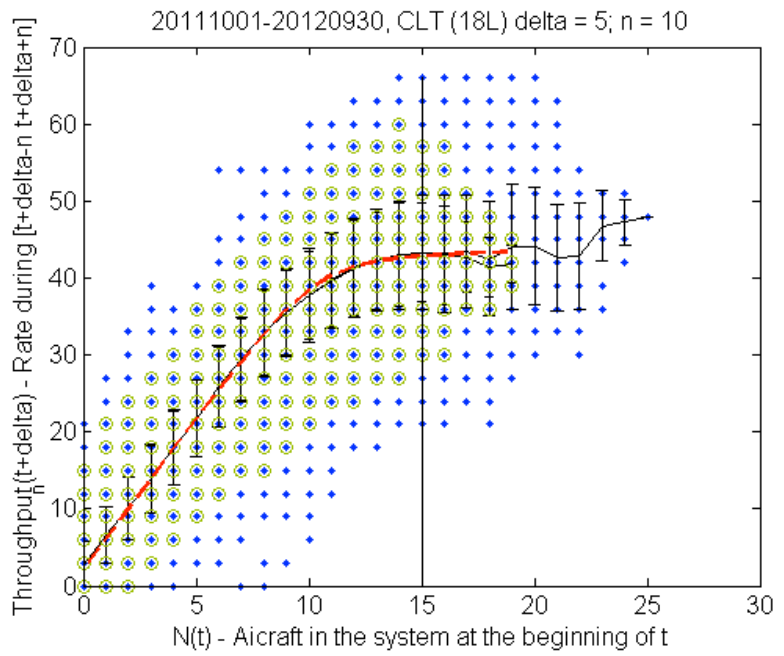

Fig. 2. Throughput saturation analysis (CLT, Runway 18L departures)

The throughput plotted on the vertical axis was measured at every minute $t$ as the number of flights that took off in a time window symmetrically centered on $t$. The time window was set to twenty minutes in this analysis (the variable $n$ in the plot). The throughput measurement was plotted at a time offset (the variable delta in the plot) from the measurement of $\mathrm{N}$. The offset was selected for each plot as zero, five, or ten minutes. The offset that resulted in the best correlation between $\mathrm{N}$ and the throughput was selected. In the example in Fig. 2 the offset (delta) is shown in the title of the plot as five minutes. The throughput is reported per hour.

The average throughput is computed at each $\mathrm{N}$ value and connected with a solid line in the plot. Error bars show the variation in throughput at each $\mathrm{N}$ value. As shown in Fig. 2, the average throughput increases and then saturates as $\mathrm{N}$ increases. In order to identify and quantify the saturation of the throughput at high $\mathrm{N}$ values, a hyperbolic curve was fitted to the average throughput versus $\mathrm{N}$ data (Dashed red line in Fig. 2 ). The fitted hyperbolic curve has two asymptotes: one horizontal asymptote that tends towards a constant throughput value as $\mathrm{N}$ tends to infinity and a second asymptote that passes through the initial point at zero N. The resulting horizontal asymptote approximates the average throughput capacity of the runway. Before performing the curve fit, the least frequent $\mathrm{N}$ - throughput pairs were eliminated as outliers representing rare and off-nominal conditions. The removed pairs are the blue dots that are not encircled in Fig. 2 and constituted in this case one percent of all pairs. The remaining pairs (green circles in the figure) were the only ones used in the curve fitting. As seen in the plot, the filtering excluded pairs of low throughput at high queue values. These pairs represent off-nominal conditions, such as airport closure or bad weather events, where high queues accumulated due to lack of throughput. The filtering also excluded pairs of high throughput at all $\mathrm{N}$ values. These pairs represent rare reports of high runway utilization.

In this paper, the $\mathrm{N}$ value that is sufficient for throughput saturation was considered at the point where the slope of the hyperbolic fit curve is 0.005 . In Fig. 2 a vertical line is drawn at this point to indicate the value $\mathrm{N}$ on the horizontal axis that corresponds to throughput saturation. In this case fifteen flights between the gate and the runway were considered sufficient to saturate the runway throughput. To study the strategic scheduler sensitivity to this saturation threshold, $75 \%$ and $50 \%$ of the saturation value were analyzed as well.

\section{B. Tactical Scheduler}

The tactical scheduler generates a runway operations schedule, which is used to simulate the runway operations. This schedule is assumed to be shared with the strategic scheduler described above, which uses the schedule as an input in the metering process. The tactical scheduler inserts departures and runway crossings in a FCFS order between a given arrival schedule. For example, departures and runway crossings on runway $18 \mathrm{C}$ at CLT are inserted in gaps between the arrivals on runway $18 \mathrm{C}$ and in gaps between the arrivals on runway 23 , which converges on runway $18 \mathrm{C}$. The scheduler does not change the given arrival schedule where arrivals are assumed to land at their actual landing times reported in the PDARS/ASDE-X data base. In addition to the landing times, the scheduler takes as input the takeoff and runway crossing ready times for each departure and crossing (respectively) in the schedule horizon, and the required time separation between successive operations. The algorithm is described first followed by the statistical models of the separation times and the unimpeded transit times used to generate the ready times.

\section{1) Scheduling algorithm}

The tactical scheduler algorithm first ranks the departures and runway crossings in the scheduling horizon according to their ready time, to takeoff or to cross the runway respectively. Then it applies the following steps to each flight according to this FCFS order:

1. Find the gaps between successive arrivals within which the ready time (to takeoff or to cross) lies. For example, for runway $18 \mathrm{C}$, there may be two gaps, one between two successive arrivals on the same runway $18 \mathrm{C}$ and one between two successive arrivals on the dependent runway 23 .

2. Find the separation required behind the leading arrival of each of the gaps. Identify the maximum of the leading arrival times plus the required separations as an upper limit on the desired schedule. 
3. Find the departures and runway crossings that were already scheduled within the arrival gaps, if any. Identify the required separation behind the last departure operation and behind the last crossing. Identify the maximum of their scheduled times plus their corresponding required separations as another upper limit on the desired schedule.

4. Compare the limiting times computed in steps 2 and 3 and set the candidate schedule to the maximum of the two.

5. Find the required separation before the trailing arrival of each arrival gap and identify the minimum of the trailing arrival times minus the required separations as a lower limit on the desired schedule.

6. If the candidate time computed in step 4 violates the lower limit computed in step 5, identify the following arrival gaps and repeat steps 1 through 6 until a gap is found where the lower limit is not violated. Once found set the operation schedule at that time.

Note 1: The algorithm described is not guaranteed to produce an optimal schedule in terms of maximum throughput or minimum delay because of two reasons: (1) The algorithm schedules flights in a FCFS sequence and a flight is not visited more than once. (2) The algorithm ignores the triangular inequality which is characteristic of the separation requirements. If three flights $\mathrm{A}, \mathrm{B}$, and $\mathrm{C}$ are operated successively, the triangular inequality implies that the separation required between $\mathrm{A}$ and $\mathrm{C}$ is larger than the sum of the separations required between $\mathrm{A}$ and $\mathrm{B}$ and between $\mathrm{B}$ and C. In this case, ensuring that $\mathrm{C}$ is separated from its immediately leading flight $\mathrm{B}$ is not sufficient to ensure that it is separated from the preceding flight $\mathrm{A}$. The algorithm described above only tests the separation from the immediately leading flight and the immediately trailing flight.

Note 2: The algorithm represents nominal operations. It is possible to space the flights by more than the minimum requirements if the capacity is reduced below nominal, by imposing a rate limit within a sliding time window.

\section{2) Runway service model}

One of the inputs to the tactical scheduler is the required separation between successive runway operations. These separations were derived using historical data analysis of the landing, takeoff and runway crossing times reported in the ASDE-X database over one year. Statistical distributions were derived for all relevant pairings of successive operations: departure-departure, crossing-crossing, crossing-departure, arrival-departure, and crossing-arrival. Different models were generated for different consecutive aircraft weight classes of arrivals and departures (for example, small behind heavy or B757, small behind large and large behind large) to capture the effect of wake turbulence. Different models were also generated for the different runway pairs (for example, departure on $18 \mathrm{C}$ behind an arrival on $18 \mathrm{C}$ versus behind an arrival on 23). Different models were also generated for consecutive runway crossings from the same crossing point or from independent crossing points, because crossings from independent crossing points can occur simultaneously.

Fig. 3 shows an example of a distribution of the separation time between two departures of the weight class large on the same runway. To reduce the effect of lulls and outliers, only operations under queuing were used to derive the required separation distributions. In addition, for each distribution the least frequent (five percent) separations were removed. The effects of these filters are the difference between the top and bottom plots in Fig. 3.
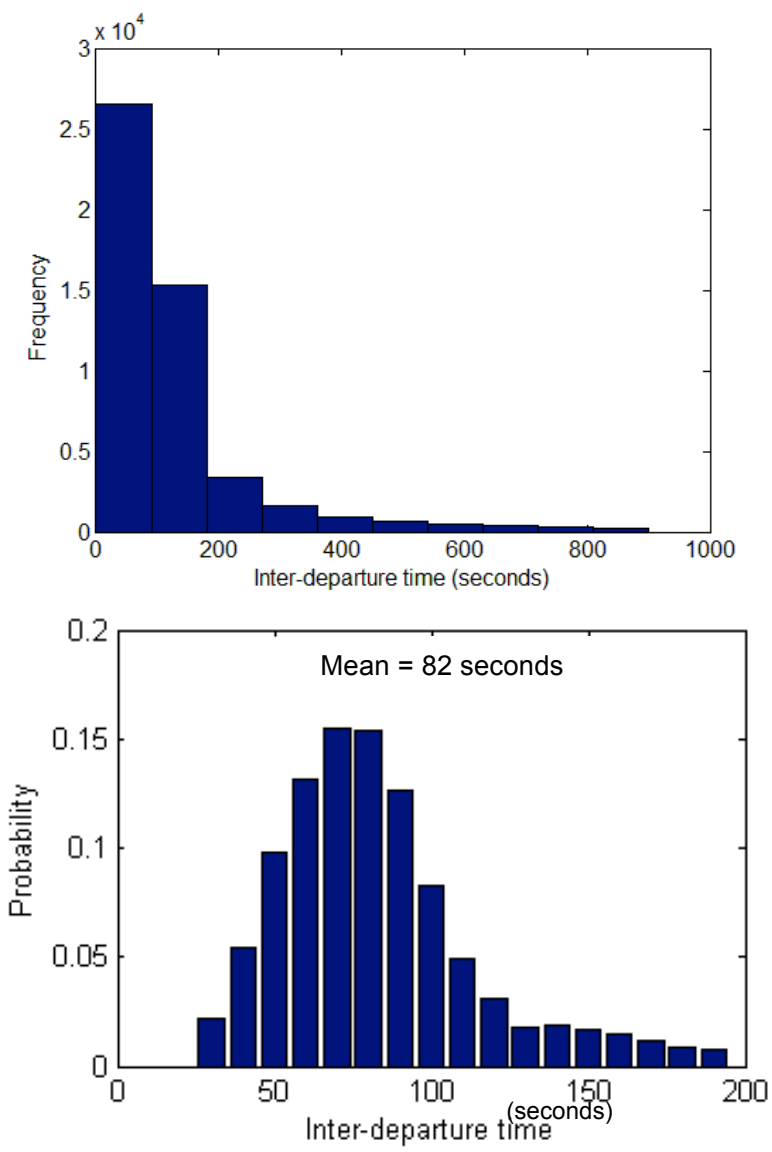

Fig. 3. Distribution of separation between two successive departures of weight class large on CLT runway 18L (top: Histogram of times below 15 minutes; bottom: distribution after filtering)

The distributions were used in two cases in the simulation: (1) The tactical scheduler used the means of the distributions for generating a runway operations schedule within the scheduling horizon. (2) The traffic progress over a simulation time step used random sampling from the distributions to introduce an uncertain deviation from the expected schedule generated in (1). In the deterministic baseline, the simulation update used the means of the distributions to be consistent with the expected schedule generated in (1).

\section{3) Unimpeded transit model}

The unimpeded transit time of a flight from the gate to the runway and from the ramp spot to the runway is used for estimating the time a flight is ready for takeoff. The unimpeded transit time from landing to the runway crossing point is also 
used to estimate the time a flight is ready to cross the runway. These ready times are used by the tactical scheduler to schedule the runway operations and by the strategic scheduler to estimate the number of flights that are ready for takeoff but did not takeoff yet. Statistical models of the unimpeded transit time were generated from one year of historical data. ASDE-X provided the landing time, the runway crossing time, the ramp exit time, and the takeoff time of each flight, in addition to the runway that was used by the flight. ASPM provided the pushback time of each flight. Details of the derivation of these models were described in a predecessor paper [27].

Distributions were generated for the transit from the ramp or the gate to the runway for each pair of airline (to represent different ramp areas) and runway. Fig. 4 shows an example of the unimpeded transit model from the ramp to the runway for one airline. Distributions were also generated for each pair of landing runway and runway crossing point. Fig. 5 shows an example of the unimpeded transit model from the landing to the runway crossing.
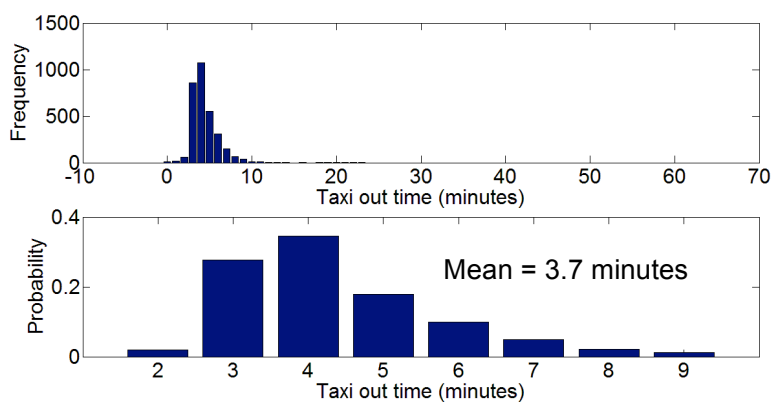

Fig. 4. Distribution of unimpeded transit from ramp to runway $18 \mathrm{~L}$ for one airline (top: histogram before filtering; bottom: distribution after filtering)
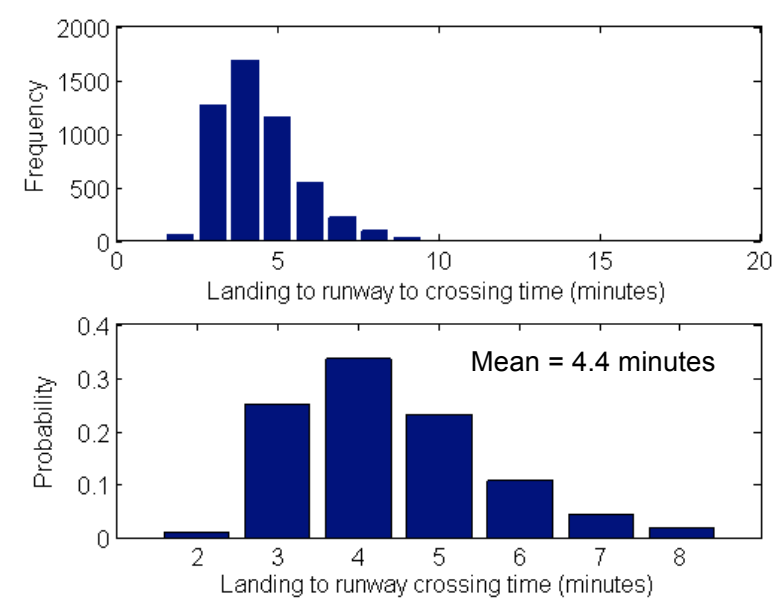

Fig. 5. Distribution of unimpeded transit from landing runway $18 \mathrm{~L}$ to crossing runway $18 \mathrm{C}$ for one crossing point (top: histogram before filtering; bottom: distribution after filtering)

To reduce the effect of lulls and outliers, only operations when queues were present were used to derive the transit time distributions [27]. In addition, for each distribution the least frequent five percent was removed. To show the effect of filtering the top of each figure shows the histogram of the data before filtering and the bottom shows the probability distribution after filtering.

The distributions were used in two cases in the simulation: (1) The tactical and strategic schedulers used the means of the distributions for scheduling runway operations and gate/ramp release times within the scheduling horizon, respectively. (2) The traffic progress over a simulation time step used random sampling from the distributions to introduce an uncertain deviation from the expected unimpeded transit used in (1). In the deterministic baseline, the simulation update used the means of the distributions to be consistent with the expected unimpeded transit times used to generate the schedule in (1).

\section{Simulation Update Cycle}

The strategic scheduler was called dynamically every fifteen minutes with a horizon that extended for the rest of the day. The tactical scheduler was also called every fifteen minutes because the analysis focused on the performance of the strategic scheduler and no effects were measured for running the tactical scheduler several times during every strategic period. The tactical scheduler horizon also extended for the rest of the day to be consistent with the strategic scheduler horizon. During a fifteen minute increment, the simulation performed the following tasks:

1. It pushed back all flights whose pushback time was due. The flight's actual pushback time (OUT time recorded in ASPM) was used to indicate the flight's readiness for pushback and hence a flight was never pushed back prior to this time. The following cases were considered: (a) If a flight was not metered (not delayed by the metering algorithm) or was metered and its pushback time assigned by the metering algorithm was less than its actual pushback time, then it was pushed back at its actual pushback time. (b) If a flight was metered and its pushback time assigned by the metering algorithm was larger than its actual pushback time, then it was pushed back at its metered pushback time. Note that if the flight plan pushback time was used to estimate demand, a metered flight may be delayed by metering relative to its flight plan pushback time but not delayed relative to its actual pushback time (which in this case is larger than the flight plan pushback time). In this case the flight was released at its actual pushback time, emulating a delay in the flight's readiness for pushback.

2. It released from the spot all flights whose ramp exit time was due. The logic is the same as described in step 1, using the actual ramp exit time recorded in the ASDE-X database instead of the actual pushback time and the ramp exit time assigned by metering instead of the pushback time assigned by metering. A flight plan ramp exit time was estimated by adding an unimpeded transit time from the gate to the taxi spot to the flight plan pushback time.

3. It added an unimpeded transit time for the flights that did not takeoff yet to estimate their ready for takeoff time. The transit time is from the ramp to the 
runway for flights that exited the ramp and from the gate to the runway for flights that did not exit the ramp. In deterministic scenarios, the simulation used the means of the unimpeded transit time distributions. In scenarios modeling transit time uncertainty, the simulation sampled transit times from the unimpeded transit time distributions.

4. It updated the takeoff times of the flights within the simulation increment by running the tactical scheduler given the takeoff ready times updated in step 3. This step simulates actual runway operations. In deterministic scenarios, the simulation used the means of the distributions of the separation times between successive runway operations. In scenarios modeling runway operations uncertainty, the simulation sampled separation times from the separation time distributions.

5. Finally, the simulation removed flights whose takeoff time (computed in step 4) was due.

\section{ANALYSIS AND RESULTS}

The fast time simulation analysis was applied to one day, July 17, 2012 at CLT. The day was selected as a typical busy day with 759 departures, 751 arrivals and considerable queuing. On this day CLT operated in the south configuration landing runway $18 \mathrm{~L}, 18 \mathrm{C}$ and 23 and departing runways $18 \mathrm{C}$ and $18 \mathrm{~L}$. Landings on runway $18 \mathrm{R}$ cross runway $18 \mathrm{C}$ on the way to the ramp. In the following subsections, we present a series of analyses using the scenarios outlined in Table 1.

TABLE I. ANALYSIS SCENARIOS

\begin{tabular}{|l|c|c|c|c|}
\hline Scenario & $\mathbf{1}$ & $\mathbf{2}$ & $\mathbf{3}$ & $\mathbf{4}$ \\
\hline Metering & Off & On & On & On \\
\hline Demand Uncertainty & Off & Off & On & Off \\
\hline $\begin{array}{l}\text { Transit \& Runway } \\
\text { Separation Uncertainty }\end{array}$ & Off & Off & Off & On \\
\hline Figures & 6 & $7-12$ & $13-14$ & $13-14$ \\
\hline
\end{tabular}

First we present a validation of the simulation model by comparing its baseline performance to the actual operations on that day. The baseline used for the validation is the first scenario in Table 1, where metering and all uncertainties are turned off. Then we analyze the impact of metering using the three metering strategies by comparing the deterministic second scenario to the deterministic first scenario in Table 1. In the second scenario metering is turned on but the uncertainties are turned off as in the first scenario. Finally, we present two analyses that compare the performance of the three metering strategies under uncertainty scenarios: In the third scenario we investigate the effect of departure demand uncertainty alone and in the forth scenario the effect of transit and service time uncertainties, with demand uncertainty turned off. Three values of the queuing parameter thresholds were used for each analysis: the value that corresponded to the throughput saturation threshold (see Fig. 2), 75\% of this value and $50 \%$ of this value, for each runway.
The performance of the metering control strategies were measured using the following metrics highlighting the tradeoff between efficiency and throughput:

1. The reduction in the congestion level due to metering measures increased efficiency.

2. The delay that was absorbed at the gate due to metering, which corresponds to the reduction in congestion, also measures increased efficiency.

3. The change in the flight takeoff time due to metering measures the overall delay and the conformance to the given runway schedule computed by the tactical scheduler. Hence it also measures the impact on runway throughput.

As explained in each of the following subsections, the metrics are measured between the two scenarios compared. These scenarios are either two scenarios from Table 1 or a scenario from Table 1 compared to a corresponding baseline that differed from the scenario only by turning metering off (i.e., no strategic scheduler).

\section{A. Baseline Model Validation}

Fig. 6 compares the performance of the model relative to the actual operations of July 17, 2012. It plots the actual number of flights that pushed back for runway $18 \mathrm{~L}$ but did not take off at each five-minute increment. It also plots the corresponding number that was estimated by the baseline simulation of scenario 1 in Table 1.

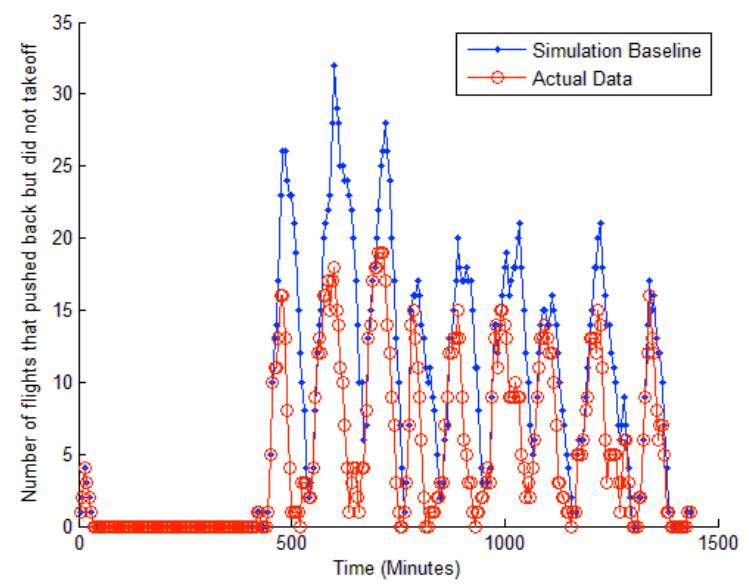

Fig. 6. Actual and simulated baseline operations of one day

The baseline scenario 1 did not apply any metering; rather flights were released from the gate and ramp according to their actual pushback and ramp exit times reported in the historical data (ASPM and ASDE-X respectively). Scenario 1 is also deterministic as all the uncertainties were turned off. Turning demand uncertainty off meant that the demand was estimated using the actual pushback, ramp exit, and landing times. The means of the unimpeded transit time distributions were added to these times to estimate the takeoff ready time and the runway crossing ready time of each flight. Turning runway operations and transit time uncertainties off meant that no 
deviations were added between the runway separation times and unimpeded transit times that were assumed by the tactical and strategic schedulers and the times that materialized in the simulation update.

Fig. 6 shows a reasonable match between the actual and simulated levels of congestion over the course of the day, with some overestimation by the model particularly during the earlier departure banks. The model performance can be improved by calibrating the separation and transit time distributions. For example, the mean of the inter-departure time distribution shown in Fig. 3 may be reduced by removing more of the tail. This would increase the modeled runway service rate and hence reduce the resulting congestion in the model. However, since the analysis reported in this paper only demonstrates mechanism effects (rather than reports validated benefits) such calibration was not conducted completely for this analysis and will be performed in future research for estimating validated benefits.

\section{B. Impact of metering on congestion in deterministic Scenario}

In this section, the deterministic baseline described in the previous section (scenario 1 in Table 1) is compared to the corresponding deterministic metering scenario (scenario 2 in Table 1), which only differed from scenario 1 by applying metering. The uncertainties were all turned off. Fig. 7, 8 and 9 show the impact of metering on queuing by comparing the queuing parameters under the baseline that does not apply metering and under the three control strategies. Fig. 7 plots on the vertical axis the number of flights that pushed back from the gate for runway $18 \mathrm{~L}$ but did not take off at each fiveminute increment of the day. Fig. 8 plots on the vertical axis the number of flights that left the ramp but did not takeoff at each five-minute increment. Finally, Fig. 9 plots on the vertical axis the number of flights that spent their unimpeded transit time to the runway but did not take off at each five-minute increment. Each figure contains four plots: one for the baseline that did not apply metering and one for each of the three control strategies, applied at $75 \%$ of the throughput saturation threshold as an example.

The three figures show that the congestion and queuing has been reduced by all the control strategies relative to the baseline. However, the queuing parameter that is displayed on the vertical axis in each of the figures was maintained at a stable level only by the method that explicitly controlled it. On the other hand, the parameter was more variable under the other methods that implicitly controlled it. Namely, as shown in Fig. 7, the number of flights that pushed back but did not take off was controlled at a stable desired value of eleven flights by the gate-to-runway control strategy that explicitly controlled this parameter. It was more variable under the other two strategies that controlled either the number of flights that exited the ramp but did not take off (spot-to-runway control) or the number of flights that spent their unimpeded transit time to the runway but did not take off (ready-to-runway control). Similarly, the number of flights that exited the ramp but did not take off in Fig. 8 was controlled at a stable level of five flights only by the spot-to-runway control method. And finally, the number of flights that spent their unimpeded transit time to the

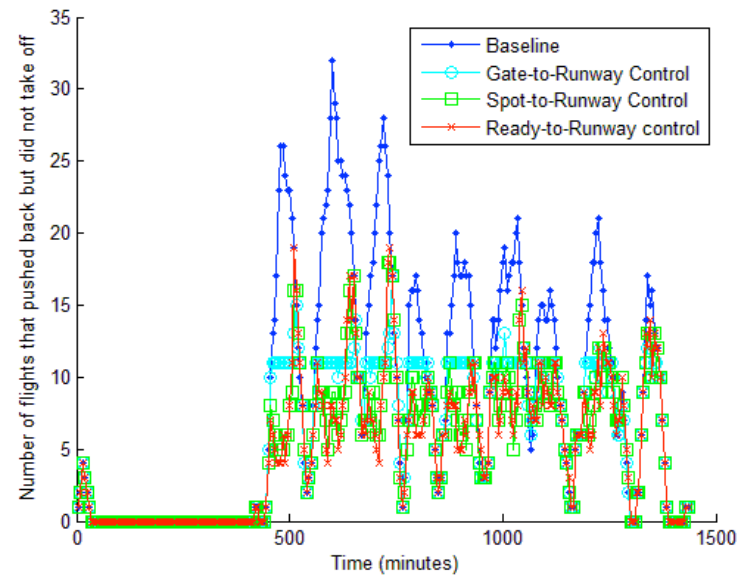

Fig. 7. Metering impact on the number of flights that pushed back but did not takeoff

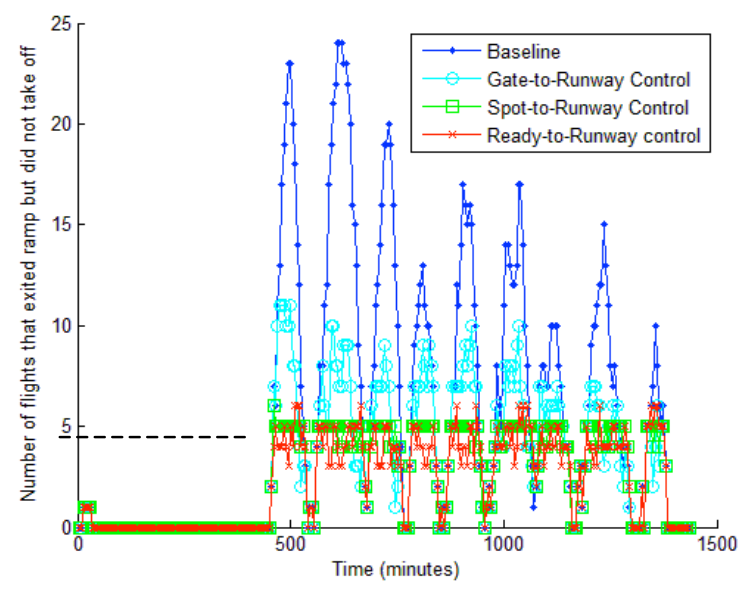

Fig. 8. Metering impact on the number of flights that exited the ramp but did not takeoff

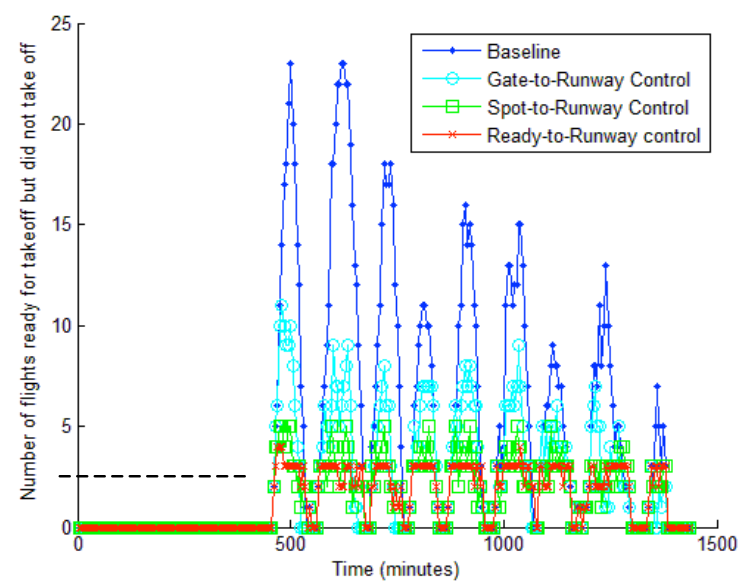

Fig. 9. Metering impact on the number of flights that were ready to takeoff but did not takeoff

runway but did not take off was controlled at a desired level of three flights only by the ready-to-runway control method. 
One can observe that occasionally, the parameter that was controlled at a stable level in each of the figures spiked above the stable level which was maintained most of the time. For example, the number of flights that pushed back but did not take off in Fig. 7, increased to thirteen flights around the minute 1000. The reason for this violation of the desired queuing parameter threshold is the conformance to the runway schedule constraint. As was mentioned in the metering algorithm description (step 3), a flight is released from the gate such that it can transit unimpeded to the runway before its scheduled takeoff time, even if the desired queuing threshold is violated. The gate occupancy constraint (step 4 of the metering algorithm) can produce the same effect, where a flight is released before causing arrivals to wait for their gate, at the expense of violating the queuing threshold. However, in this analysis the gate capacity of 97 gates, shared by all airlines, was sufficiently high that the constraint was not invoked. Future research will investigate variations on the gate capacity assumptions made in this paper.

Fig. 10 compares the average values for each of the queuing parameters in Fig. 7-9, over the busy period of the day (between 500 and 1400 minutes). The figure shows that all of the metering strategies reduced the average congestion relative to the baseline without metering, as was evident in Fig. 7-9. The mean values in Fig. 10 are lower than the thresholds depicted in Fig. 7-9, because the thresholds are used by the metering algorithm as maximum target values (except when runway conformance or gate capacity constraints necessitate otherwise) and the averaging period included high and low departure demand.

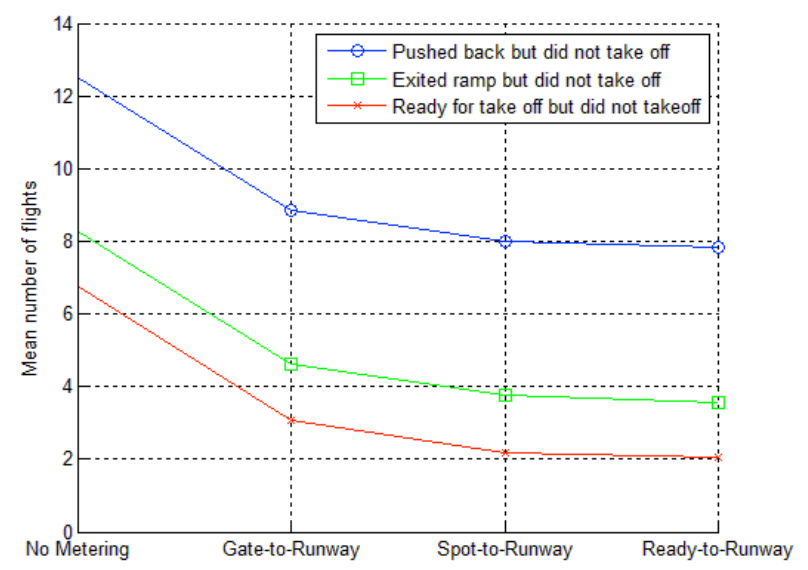

Fig. 10. Average queuing under different metering strategies

Fig. 10 also shows that the metering method that controlled the number of flights that pushed back but did not take off (gate-to-runway) resulted in higher mean values than the other two strategies for all of the three queuing parameters, in the deterministic scenario. This indicates a clear dependence of the congestion and queuing levels, measured by any of the three queuing parameters, on the size of the system being controlled.

\section{Impact of buffer size in deterministic scenario}

As mentioned in the introduction, the desire is to maximize the efficiency gains of transferring as much delay to the gate as possible while minimizing the impact on runway throughput. Fig. 11 compares the performance of the three metering strategies in terms of the average delay absorbed at the gate because of the metering. It is followed by Fig. 12, which shows the corresponding average increase in the takeoff time under each strategy. Each figure shows these averages for the three threshold values of the queuing parameters: the value that corresponded to historical throughput saturation, $75 \%$ of this value and $50 \%$ of this value.

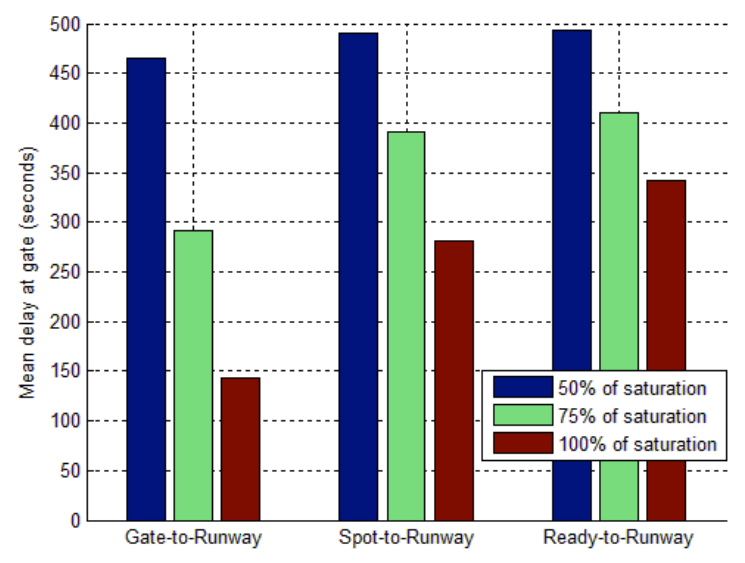

Fig. 11. Gate delay using different control strategies under deterministic scenario

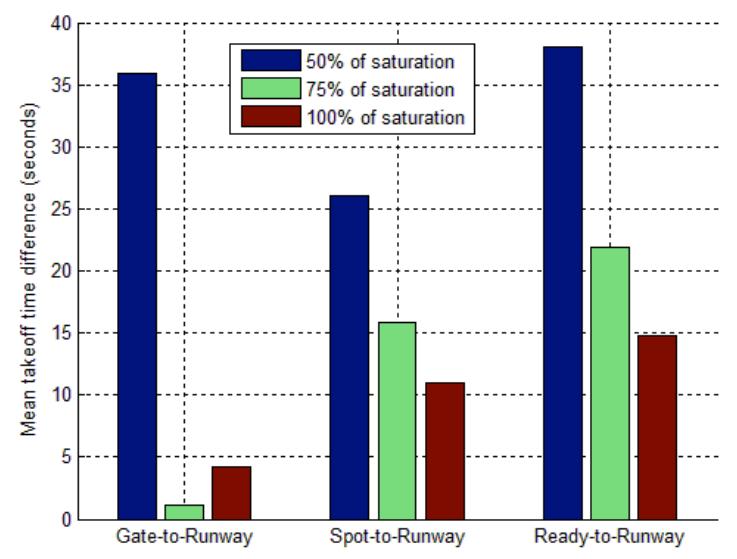

Fig. 12. Takeoff time difference using different control strategies under deterministic scenario

Fig. 11 shows that for each of the metering strategies, the average delay absorbed at the gate was larger using the smaller queuing parameter thresholds as expected because more delay at the gate is needed to maintain smaller queuing buffers. Fig. 12 demonstrates that the larger amount of delay that is transferred to the gate occurs at the expense of delayed takeoff time. This tradeoff is clear for all the strategies. For example, using gate-to-runway control with the smallest queuing parameter threshold at $50 \%$ of the saturation level caused an average takeoff delay of 36 seconds. However, with the 
queuing parameter threshold set at the level needed for throughput saturation ( $100 \%$ of saturation), this takeoff time difference is negligible at less than five seconds. To achieve this low takeoff time delay however, a smaller average metering delay of 150 seconds (2.5 minutes) is possible.

The gate-to-runway control strategy performed better than the other two strategies in terms of maintaining small takeoff time difference as evident from Fig. 12. As mentioned above, at the higher queuing parameter thresholds of $75 \%$ to $100 \%$ of the throughput saturation level, it was possible to maintain the takeoff time difference below five seconds with the gate-torunway control strategy, with an average metering delay at the gate of 150 seconds. The spot-to-runway and ready-to-runway control strategies, respectively resulted in takeoff time differences of ten and fifteen seconds at the throughput saturation levels and metering gate delay of 275 and 350 seconds. This indicates that these strategies are less effective at maintaining throughput using the thresholds derived from the historical data analysis. Either larger queuing parameter thresholds should be used - ones that reduce the takeoff time difference to near zero, or they should be combined with gateto-runway control elements to maintain sufficient flights in the system to ensure throughput saturation.

\section{Impact of uncertainty}

The two non-deterministic scenarios in Table 1 are analyzed in this section: scenario 3 adds uncertainty in the demand and scenario 4 adds uncertainty in the transit time and runway separations (runway service rate) to the deterministic scenario described in the previous section. In scenario 3 , the schedulers used the pushback times published in the flight plans instead of the actual pushback times, when estimating the demand (i.e., takeoff ready times) of flights that have not pushed back yet. To estimate the demand for the runway the modeled unimpeded transit time was added to the pushback time (for flights that did not exit the ramp yet) and to the ramp exit time (for flights that exited the ramp). No uncertainty was modeled in the arrival demand as the actual landing times reported in ASDE-X/PDARS were used for all cases. Otherwise the simulation is identical to the one used in the previous section. The schedulers and the simulation update used the means of the unimpeded transit time and separation time distributions to be consistent with each other. In scenario 4 , the schedulers used the means of the unimpeded transit time and the separation time distributions when scheduling flights. On the other hand the simulation update during each time increment used random sampling from these distributions to introduce an uncertain deviation between the progress of a flight and its desired schedule. In this scenario the demand was known perfectly by the schedulers and the simulation update used the actual pushback and ramp exit times rather than the flight plan schedule.

The analysis of the previous section showed that the queuing parameter threshold that corresponds to the historical throughput saturation is needed to maintain runway throughput even in the deterministic case. Therefore, we use only this threshold in the analysis of the impact of uncertainty in this section. The threshold values that are smaller than this threshold resulted in similar trends but inferior performance.
Fig. 13 compares the mean gate delay that was imposed by the three metering strategies between the two uncertainty scenarios and the deterministic scenario from Fig. 11. Fig. 14 compares the corresponding takeoff time delays.

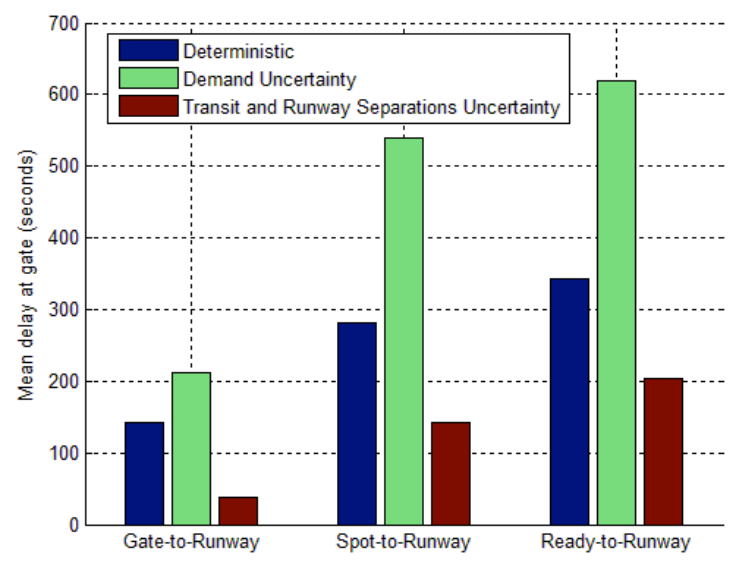

Fig. 13. Gate delay using different control strategies under uncertainty

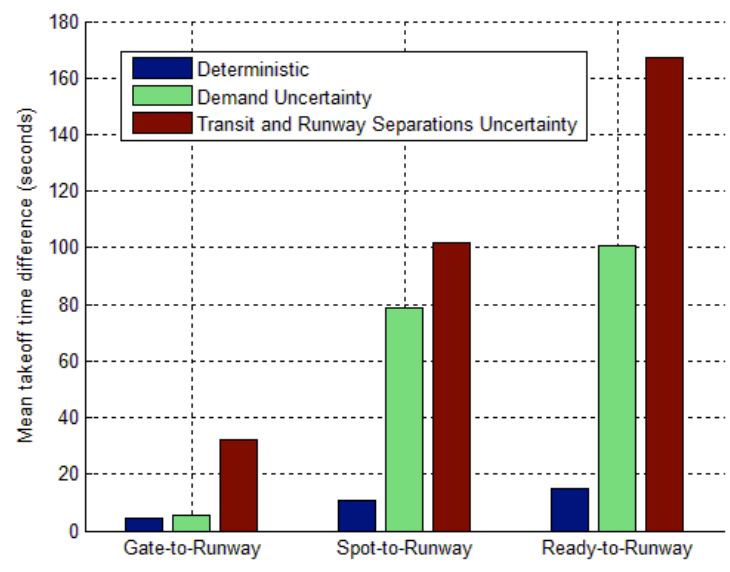

Fig. 14. Takeoff time difference using different control strategies under uncertainty

\section{1) Impact of demand uncertainty}

Fig. 14 shows that the uncertainty in the demand increased the takeoff time delay significantly (by almost ten times in the spot-to-runway and ready-to-runway control strategies) compared to the deterministic scenario. The gate-to-runway control strategy with the queuing parameter threshold set at $100 \%$ of the throughput saturation level is the only strategy that was able to reasonably handle the demand uncertainty; it kept the takeoff time delay small at about eight seconds. The corresponding gate delay was also significant at about 200 seconds for this case as shown in Fig. 13. This gate delay value is significantly smaller than the gate delay that was applied by the other two control strategies. Since larger gate delay leads to larger takeoff time delay, this explains the smaller takeoff time delay that resulted under the gate-to-runway control strategy. 


\section{2) Impact of transit time and runway separation uncertainties}

Fig. 14 shows that the uncertainty about the transit and runway service times increased the takeoff time delay in all of the metering strategies compared to the deterministic scenario. The increase was greater than the one due to the uncertainty in the demand, with a lowest takeoff time delay of about 35 seconds under the gate-to-runway control strategy. This may be due in part to the shorter time available to adjust to the uncertainties in the transit time and runway separations relative to the uncertainty in the pushback time (demand). An interesting observation is that the gate delay applied by all the metering strategies was smaller under the transit time and runway separations uncertainty compared to under demand uncertainty and the deterministic scenario, as evident from Fig. 13. For example, the gate-to-runway metering strategy delayed flights at their gate only forty seconds on average leading to 35 -second average takeoff time delay. Therefore, under these uncertainties, the metering strategies are much less effective at transferring delay to the gates without impacting the runway schedule or throughput. Hence, further research is needed to devise more robust metering approaches to handle such uncertainties.

\section{CONCLUDING REMARKS}

We presented a simulation-based analysis that compared the performance of different control strategies for departure metering, as part of an integrated departure scheduling concept. The performance was compared in terms of the ability of the different strategies to conform to a runway schedule and hence maintain runway throughput while transferring queuing delays from the airport movement area to the gate to save on fuel burn and emissions. The following observations are made:

A control strategy that applied metering by maintaining the number of flights that pushed back but did not takeoff was more effective than the strategies that controlled the number of flights that exited the ramp but did not takeoff or controlled the number of flights that spend their unimpeded transit time to the runway but did not takeoff. This was true both in a deterministic case and in cases with uncertainties about the demand, the transit time, and the runway service times. This observation indicates the need to maintain flights throughout the system between the gates and the runway rather than just at the runway end or between the ramp and the runway, to ensure continuity in traffic supply to the runway.

The best performing metering strategy that controlled the number of flights that pushed back but did not take off was able to handle the demand uncertainty modeled in this analysis, keeping the runway throughput high while delaying flights at their gate by an average of 2.5 minutes. However, under transit time and runway service uncertainties, a small gate delay impacted the runway throughput significantly even with the largest queuing parameter threshold.

It should be noted that these observations may not generalize to other scenarios of uncertainties that were not analyzed in this paper. Further research is being conducted to investigate the performance under additional scenarios of uncertainties and with alternative more robust metering strategies such as using a combination of the three strategies analyzed in this paper. Future research will also investigate different interactions between the strategic metering process and the tactical runway scheduling and schedule conformance processes. Finally, the application of these concepts will be studied at other airports and configurations.

\section{REFERENCES}

[1] Idris H., "Identification of Local and Propagated Queuing Effects at Major Airports," Presented at the AIAA Aviation Forum, Aviation Technology, Integration and Operations (ATIO) conference, Dallas, TX, July 2015.

[2] Swenson H., Thipphavong J., Sadovsky A., Chen L., Sullivan C., and Martin L., "Design and Evaluation of the Terminal Area Precision Scheduling and Spacing System." Ninth USA/Europe Air Traffic Management Research and Development Seminar (ATM2011), Berlin, Germany, 2011.

[3] Thipphavong J., Swenson H., Lin P., Seo A. and Bagasol A., "Efficiency Benefits Using the Terminal Area Precision Scheduling and Spacing System." AIAA Aviation Technology Integration and Operations (ATIO), Virginia Beach, VA. 2011.

[4] Hayashi M., Hoang T., Jung Y., Gupta G., Malik W., and Dulchinos V., "Usability Evaluation of the Spot and Runway Departure Advisor (SARDA) Concept in a Dallas/Fort Worth Airport Tower Simulation." Tenth USA/Europe Air Traffic Management Research and Development Seminar, Chicago, IL. 2013.

[5] Brinton C., Provan C., Lent S., Prevost T., and Passmore S., "Collaborative Departure Queue Management," Ninth USA/Europe Air Traffic Management Research and Development Seminar (ATM2011), Berlin, Germany. 2011.

[6] Simaiakis I., Khadilkar H., Balakrishnan H., Reynolds T., Hansman R.J., Reilly B., and Urlass S., "Demonstration of Reduced Airport Congestion Through Pushback Rate Control," Ninth USA/Europe Air Traffic Management Research and Development Seminar (ATM2011), Berlin, Germany. 2011.

[7] Engelland, S., Capps, A., Day, K., "Precision Departure Release Capability (PDRC) Concept of Operations," NASA/TM-2013-216534, June 2013.

[8] Engelland S. and Capps A., "Trajectory-Based Takeoff Time Predictions Applied to Tactical Departure Scheduling: Concept Description, System Design, and Initial Observations," 11th AIAA Aviation Technology, Integration, and Operations (ATIO) Conference, 2011.

[9] Gupta G., Malik W., and Jung Y., "Incorporating Active Runway Crossing in Airport Departure Scheduling." AIAA Guidance, Navigation, and Control Conference, Ontario, Canada, 2010.

[10] Capps A., Kistler M. and Engelland S., "Design Characteristics of a Terminal Departure Scheduler," 14th AIAA Aviation Technology, Integration, and Operations (ATIO) Conference, 2014.

[11] Zelinski S., "A Framework for Integrating Arrival, Departure and Surface Operations Scheduling," 33rd Digital Avionics Systems Conference (DASC), October 5-9, Colorado Springs, CO, 2014.

[12] Callantine T., Kupfer M., Martin L, Mercer J., and Prevot T, SystemLevel Performance Evaluation of ATD-1 Ground-Based Technologies. 14th AIAA Aviation Technology, Integration, and Operations (ATIO) Conference, Atlanta, GA, 2014.

[13] Xue M. and Zelinski S., "Dynamic Stochastic Scheduler for Integrated Arrivals and Departures", 33rd Digital Avionics Systems Conference (DASC), October 5-9, Colorado Springs, CO, 2014.

[14] Bosson C., Xue M., and Zelinski S., "Optimizing Integrated Terminal Airspace Operations Under Uncertainty", 33rd Digital Avionics Systems Conference (DASC), October 5-9, Colorado Springs, CO, 2014.

[15] Bertsimas D., "Air Traffic Flow Management at Airports: A Unified Optimization Approach," Tenth USA/Europe Air Traffic Management Research and Development 
[16] Aponso B., Coppenbarger R., Jung Y., Quon L., Lohr G., O’Conner N., and Engelland S., "Identifying Key Issues and Potential Solutions for Integrated Arrival, Departure, Surface Operations by Surveying Stakeholder Preferences," 15th AIAA Aviation Technology, Integration, and Operations (ATIO), Dallas, TX, 2015.

[17] Chevalley E., Parke B., Kraut J., Bienert N., Omar F., and Palmer E., "Scheduling and Delivering Aircraft to Departure Fixes in the NY Metroplex with Controller-Managed Spacing Tools," 15th AIAA Aviation Technology, Integration, and Operations (ATIO), Dallas, TX, 2015.

[18] Shen N., H. Idris, and V. Orlando, 2012, Estimation Of Departure Metering Benefits At Major Airports Using Queuing Analysis, 31st Digital Avionics Systems Conference, Williamsburg, VA, Oct.

[19] Idris H. and N. Shen, 2014, Impact of Gate Availability on Departure Metering Benefit, 14th AIAA Aviation Technology, Integration, and Operations (ATIO) Conference, Atlanta, Georgia.

[20] Nakahara, A., and T. G. Reynolds, An Approach for Estimating Current and Future Benefits of Airport Surface Congestion Management Techniques, 12th AIAA Aviation Technology, Integration, and Operations (ATIO) Conference, Indianapolis, Indiana, 2012.

[21] Simaiakis, I., and H. Balakrishnan, 2009, Queuing Models of Airport Departure Processes for Emissions Reduction Techniques, Proceedings of the AIAA Guidance, Navigation and Control Conference.

[22] Idris, H., A. Evans, S. Evans, and D. Kozarsky, 2004, Refined Benefits Assessment of Multi-Center Traffic Management Advisor for Philadelphia and New York, 4th AIAA Aviation Technology, Integration, and Operations (ATIO) conference, Chicago, IL.

[23] Evans A. and . Idris, Estimation of Arrival Capacity and Utilization at Major Airports, 6th USA/Europe Air Traffic Management Research and Development Seminar, Baltimore, Maryland, June 27 - 30.

[24] Pujet, N., 1999, Modeling and Control of the Departure Process of Congested Airports, PhD Thesis. Cambridge, Massachusetts Institute of Technology.

[25] Idris H., J-P Clarke, R. Bhuva, and L. Kang, 2002 Queuing Model for Taxi-Out Time Estimation, Air Traffic Control Quarterly Journal, Volume 10, Number 1.

[26] Saraf A., Timar S., Shen N., and Idris H., "Preliminary Queuing Analysis of Integrated departure operations in metroplex systems," 34th Digital Avionics Systems Conference, Prague, Czech Republic, September 13-17, 2015.

[27] Idris H., Shen N., Saraf A., Bertino J., and Luch N., " Queue Buffer Sizing for Efficient and Robust Integrated Departure Scheduling," 16th AIAA Aviation Technology, Integration, and Operations (ATIO) Conference, Washington, D.C., 2016.

[28] Saraf A., Bertino J., Luch N., Shen N., and Idris H., "Miles-in-Trail Restrictions Relaxation: A Key Benefit Mechanism of Integrated Arrival Departure Surface Traffic Management," 16th AIAA Aviation Technology, Integration, and Operations (ATIO) Conference, Washington, D.C., 2016.

35th Digital Avionics Systems Conference

September, 2016 